\title{
Six-month humoral response to BNT162b2 mRNA COVID-19 vaccine in people with multiple sclerosis treated with natalizumab
}

\author{
Manuela Altieri ${ }^{1} \cdot$ Rocco Capuano $^{1} \cdot$ Miriana Conte ${ }^{1}$. Giovanna Donnarumma ${ }^{2}$ Elena Grimaldi ${ }^{2} \cdot$ Nicola Coppola $^{3}$. \\ Massimiliano Galdiero ${ }^{2}$. Alessandro d'Ambrosio ${ }^{1} \cdot$ Gioacchino Tedeschi $^{1} \cdot$ Antonio Gallo $^{1}$ (i)
}

Received: 30 November 2021 / Accepted: 4 February 2022 / Published online: 16 February 2022

(c) Fondazione Società Italiana di Neurologia 2022

\begin{abstract}
Background Few studies investigated the immune response to SARS-CoV-2 vaccine in patients with multiple sclerosis (pwMS) treated with natalizumab (NTZ) and found a short-term efficient humoral response; however, there are no studies assessing the levels of SARS-CoV-2 IgG antibodies in pwMS treated with NTZ over time.

Methods Humoral immune response to BNT162b2 mRNA COVID-19 vaccine was assessed in a group of 26 pwMS on NTZ up to 6 months after a full COVID-19 vaccination cycle and compared it with 43 age- and sex-matched group of HC. Serum samples were collected before the first dose (T0), and 4 weeks (T1) and 6 months (T2) after the first dose of BNT162b2 mRNA COVID-19 vaccine. The LIAISON® SARS-CoV-2 TrimericS-IgG assay (DiaSorin-S.p.A.) was employed for the detection of IgG antibodies to SARS-CoV-2 spike protein (cutoff for positive IgG antibodies: $33.8 \mathrm{BAU} / \mathrm{mL}$ ).

Results At T1 and T2, both groups showed an efficient humoral response to BNT162b2 mRNA COVID-19 vaccine. A significant reduction of IgG antibodies to SARS-CoV-2 spike protein was detected at T2 both in pwMS and in HC, but SARS-CoV-2 IgG antibodies were still above the cutoff limit in all participants.

Conclusions pwMS on NTZ develop and maintain a long-term humoral response after a full COVID-19 vaccination cycle comparable to their healthy peers, and these findings are relevant for clinicians called to counsel about COVID-19 mRNA vaccine timing and booster doses in pwMS treated with NTZ.
\end{abstract}

Keywords COVID-19 $\cdot$ Humoral response $\cdot$ Multiple sclerosis $\cdot$ Natalizumab $\cdot$ BNT162b2 mRNA COVID-19 vaccine Vaccine

\section{Introduction}

Few studies investigated the immune response to SARSCoV-2 vaccine in patients with multiple sclerosis (pwMS) on natalizumab (NTZ) [1-3], a high-efficacy diseasemodifying therapy (DMT) that binds to $\alpha 4 \beta 1$-integrin and inhibits migration of leukocytes into the central nervous system, thus halting inflammatory activity typical of MS

Antonio Gallo

antonio.gallo@unicampania.it

1 Department of Advanced Medical and Surgical Sciences (DAMSS), University of Campania "Luigi Vanvitelli", Naples, Italy

2 Department of Experimental Medicine, University of Campania “Luigi Vanvitelli", Naples, Italy

3 Department of Mental Health and Public Medicine, University of Campania “Luigi Vanvitelli”, Naples, Italy
$[4,5]$. In the first short-term study on pwMS on NTZ [1], in which levels of IgG antibodies to SARS-CoV-2 spike protein (anti-TSP IgG) were evaluated in 31 pwMS 7 days after the second dose of the BNT162b2 mRNA vaccine, the authors reported an efficient humoral response, with values comparable to those of age- and sex-matched healthy controls (HCs). In another cross-sectional study evaluating the humoral response $\sim 3$ months after the second BNT162b2 mRNA vaccine dose in 32 pwMS treated with NTZ, a positive humoral response was measured in all pwMS, with no significant differences on levels of anti-TSP IgG between the NTZ and HC groups [2]. A third study on a larger population of 100 pwMS on NTZ confirmed that 4 weeks after the second dose of a mRNA vaccine (BNT162b2 or mRNA-1273), all participants mounted a full humoral response [3].

To date, there are no studies assessing anti-TSP IgG levels in pwMS treated with NTZ over time; thus, it is important to explore the humoral response evolution in this population 
Fig. 1 Log-transformed values of levels of antibodies to SARS$\mathrm{CoV}-2$ spike protein in people with multiple sclerosis (pwMS) and healthy controls (HC) at T0 (before the vaccination cycle), T1 (4 weeks after the first dose), and T2 (6 months after the first dose)

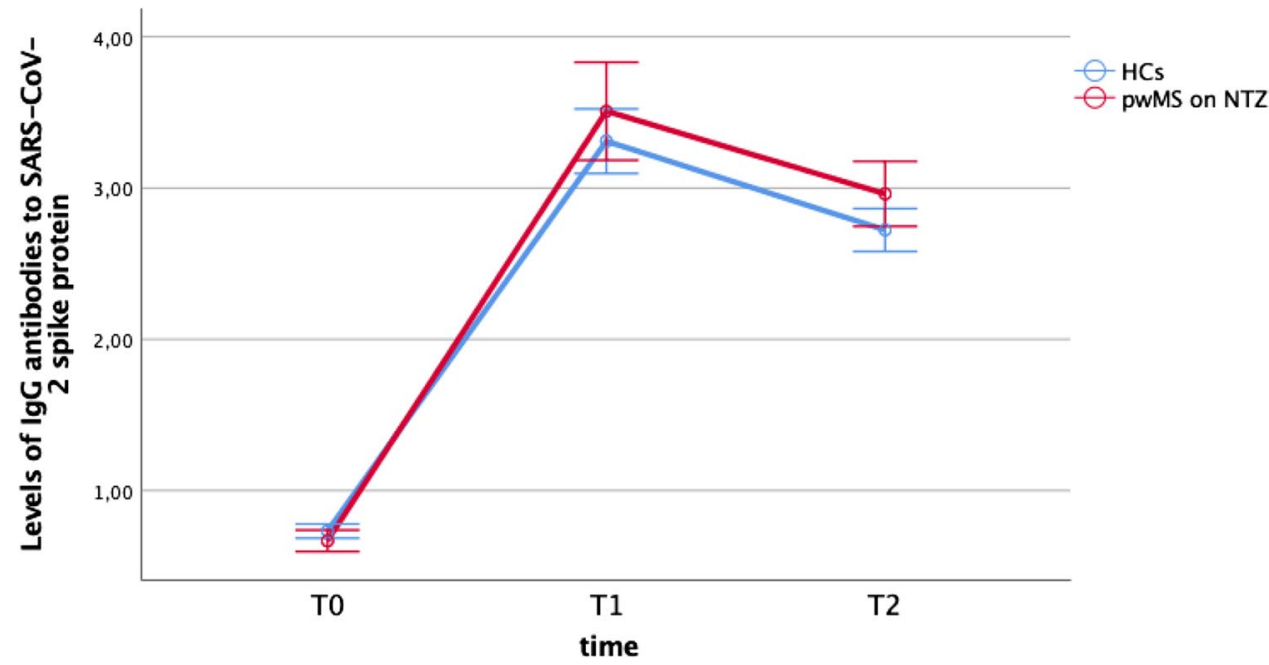

also in view of a booster dose. To address this question, we measured the humoral immune response to BNT162b2 mRNA COVID-19 vaccine in pwMS on NTZ up to 6 months after the first dose of a full COVID-19 vaccination cycle and compared it with age- and sex-matched HCs.

\section{Materials and methods}

Serum samples were collected before the first dose (T0), and 4 weeks (T1) and 6 months (T2) after the first dose of BNT162b2 mRNA COVID-19 vaccine. To be included in the study, both HCs and pwMS on NTZ did not have to have (a) a history of COVID-19, (b) positive anti-TSP $\mathrm{IgG}$ at $\mathrm{T} 0$, and (c) treatment with corticosteroids within 1 month before the first dose of vaccine. The LIAISON® SARS-CoV-2 TrimericS-IgG assay (DiaSorin-S.p.A.) was employed for the detection of anti-TSP IgG, including neutralizing antibodies [6]. The IgG titers were expressed in binding antibody units (BAU), whereas the cutoff for positive $\mathrm{IgG}$ antibodies was set at $33.8 \mathrm{BAU} / \mathrm{mL}$ [7]. Sera analysis was conducted at the virology laboratory of our University Hospital. The study was approved by the local Ethics Committee, in accordance with the Helsinki Declaration (approval code: 0015914). Each participant was informed on the aim of the study and was asked to sign an informed consent form.

Statistical analysis was performed with SPSS, version 25.0. Mean, standard deviation, median, and P25-75 were calculated to describe variables, whereas normality of distribution was assessed with Komolgorov-Smirnov test; due to the non-normality of distributions, a logarithmic transformation was performed for anti-TSP IgG values. The comparison between pwMS on NTZ and HCs on sociodemographic variables was performed with chisquare $\left(\chi^{2}\right)$ and Mann-Whitney $U$ test, as appropriate, whereas the comparison on levels of anti-TSP IgG over time was performed with a multivariate analysis of covariance (MANCOVA), with anti-TSP at T0, T1, and $\mathrm{T} 2$ as dependent variables, and the group of participants (pwMS on NTZ vs HCs) as independent variable, controlling fox sex, age, EDSS and DMT duration. Multiple regression analyses were performed to assess predictors (age, sex, EDSS, disease duration, DMT duration) of change in levels of anti-TSP IgG over time in both groups.

\section{Results}

Thirty-two pwMS on NTZ and forty-three HC-matched from a larger dataset of HCs enrolled in a surveillance program at our institution were screened; 6 pwMS were excluded from the study due to IgG titers above the cutoff at $\mathrm{T} 0$, suggesting a history of COVID-19. The final sample included 26 pwMS on NTZ and $43 \mathrm{HC}$. The sample of pwMS on NTZ (females: 69.2\%) had a median: age of 31.3 years (P25$75=24.3-41.9)$, EDSS of $1.5(\mathrm{P} 25-75=1.0-2.5)$, disease duration of 4.3 years (P25-75 $=2.7-9.5)$, and DMT duration of 1.8 years $(\mathrm{P} 25-75=0.008-7.4)$. No differences were found between pwMS on NTZ and HC on age $(U=411.00$, $Z=-1.833, p=0.067)$ and $\operatorname{sex}\left(\chi^{2}(1)=0.123, p=0.725\right)$. At T1 and T2, both groups showed an efficient humoral response to BNT162b2 mRNA COVID-19 vaccine; the MANCOVA revealed a significant main effect of time $(\Lambda=0.151, F(2,62)=174.364 ; p<0.001)$ on Anti-TSP levels; the time $*$ group interaction was not significant $(\Lambda=0.952$, $F(2,62)=1.570 ; p=0.216$; see Fig. 1$)$ : levels of anti-TSP at $\mathrm{T} 0$ were significantly lower than those at $\mathrm{T} 1 \mathrm{I}-\mathrm{J}=-2.709$, $\mathrm{SE}=0.061)$ and $\mathrm{T} 2(\mathrm{I}-\mathrm{J}=-2.142, \mathrm{SE}=0.043)$, and levels of anti-TSP at T2 were significantly lower than those at T1 $(\mathrm{I}-\mathrm{J}=-0.567, \mathrm{SE}=0.055)$. Regression analysis showed that 
EDSS significantly predicted changes in anti-TSP IgG levels over time (beta $=-0.515, t=-2.943, p=0.007$ ) in pwMS on the NTZ groups; in the HCs group, no significant predictors of change in anti-TSP IgG levels over time were found.

\section{Discussion}

The results of this study confirm and expand previous studies [1-3] by showing that pwMS on NTZ develop and maintain a 6-month humoral response after the first dose of a full COVID-19 vaccination cycle comparable to their healthy peers; moreover, we found that, in pwMS on NTZ, higher physical disability is associated with a lower humoral response over time. These findings are relevant for clinicians called to counsel about COVID-19 mRNA vaccine timing and booster doses in pwMS treated with NTZ. Of course, our study is not exempt from limitations: (a) B- and T-cell immunity was not assessed; (b) humoral response was assessed just to BNT162b2 mRNA COVID-19 vaccine; therefore, our results are not generalizable to other mRNA or viral vector vaccines; (c) our MS sample included mostly young patients with a relatively low physical disability and a short disease duration. Taken together our results provide evidence on the long-term humoral response to BNT162b2 mRNA COVID-19 vaccine in pwMS treated with NTZ, indicating that this high-efficacy DMT might not interfere with the ability to mount and maintain an efficient $\mathrm{IgG}$ response for at least 6 months.

Acknowledgements The authors thank Gabriella Andreone, Federica Giuliano, and Pasquale Sozio for helping in data acquisition and analysis.

\section{Declarations}

Conflict of interest The authors declare that there is no conflict of interest.

\section{References}

1. Capuano R, Donnarumma G, Bisecco A et al (2021) Humoral response to SARS-CoV-2 mRNA vaccine in patients with multiple sclerosis treated with natalizumab. Ther Adv Neurol Disord 14:17562864211038112. https://doi.org/10.1177/1756286421 1038111

2. Achiron A, Mandel M, Dreyer-Alster S et al (2021) Humoral immune response in multiple sclerosis patients following PfizerBNT162b2 COVID19 vaccination: up to 6 months cross-sectional study. J Neuroimmunol 361:577746. https://doi.org/10. 1016/j.jneuroim.2021.577746

3. Sormani MP, Inglese M, Schiavetti I et al (2021) Effect of SARSCoV-2 mRNA vaccination in MS patients treated with disease modifying therapies. EBioMedicine 72:103581. https://doi.org/ 10.1016/j.ebiom.2021.103581

4. Kappos L, Bates D, Hartung HP et al (2007) Natalizumab treatment for multiple sclerosis: recommendations for patient selection and monitoring. Lancet Neurol 6:431-441. https://doi.org/10. 1016/S1474-4422(07)70078-9

5. Tysabri. European Medicines Agency. https://www.ema.europa. eu/en/medicines/human/referrals/tysabri. Accessed 20 May 2021

6. Bonelli F, Blocki FA, Bunnell T et al (2021) Evaluation of the automated LIAISON® SARS-CoV-2 TrimericS IgG assay for the detection of circulating antibodies. Clin Chem Lab Med 59:14631467. https://doi.org/10.1515/cclm-2021-0023

7. Kristiansen PA, Page M, Bernasconi V et al (2021) WHO International Standard for anti-SARS- CoV-2 immunoglobulin. Lancet 397:1347-1348. https://doi.org/10.1016/S0140-6736(21)00527-4

Publisher's note Springer Nature remains neutral with regard to jurisdictional claims in published maps and institutional affiliations. 